\title{
Data-driven SIRMs-connected FIS for prediction of external tendon stress
}

\author{
See Hung Lau ${ }^{* 1}$, Chee Khoon $\mathrm{Ng}^{1 \mathrm{a}}$ and Kai Meng Tay ${ }^{2 b}$ \\ ${ }^{1}$ Department of Civil Engineering, Faculty of Engineering, Universiti Malaysia Sarawak, 94300 Kota, \\ Samarahan, Sarawak, Malaysia \\ ${ }^{2}$ Department of Electronic Engineering, Faculty of Engineering, Universiti Malaysia Sarawak, \\ 94300 Kota, Samarahan, Sarawak, Malaysia
}

(Received January 6, 2014, Revised September 9, 2014, Accepted November 3, 2014)

\begin{abstract}
This paper presents a novel harmony search (HS)-based data-driven single input rule modules (SIRMs)-connected fuzzy inference system (FIS) for the prediction of stress in externally prestressed tendon. The proposed method attempts to extract causal relationship of a system from an input-output pairs of data even without knowing the complete physical knowledge of the system. The monotonicity property is then exploited as an additional qualitative information to obtain a meaningful SIRMs-connected FIS model. This method is then validated using results from test data of the literature. Several parameters, such as initial tendon depth to beam ratio; deviators spacing to the initial tendon depth ratio; and distance of a concentrated load from the nearest support to the effective beam span are considered. A computer simulation for estimating the stress increase in externally prestressed tendon, $\Delta f_{p s}$, is then reported. The contributions of this paper is two folds; (i) it contributes towards a new monotonicity-preserving data-driven FIS model in fuzzy modeling and (ii) it provides a novel solution for estimating the $\Delta f_{p s}$ even without a complete physical knowledge of unbonded tendons.
\end{abstract}

Keywords: bond reduction coefficient; externally prestressed tendon stress; harmony search; monotonicity index; single input rule modules (SIRMs)-connected fuzzy inference system (FIS)

\section{Introduction}

Externally prestressed beam is a structural concrete member where the prestressing tendons are placed on the outside of the concrete section and are attached by anchors and deviators at discrete locations (Naaman and Alkhairi 1991a, Ng 2003). The idea of prestressing tendon placement (or sometime known as externally prestressing technique) has been growing rapidly in rehabilitating and strengthening the components of existing structure due to progressive aging and corrosion of steel reinforcement (Ariyawardena and Ghali 2002, Naaman and Alkhairi 1991a, Ng 2003). Comparing to the conventional prestressing technique (i.e., bonded tendon), externally prestressing

*Corresponding author, Ph.D., E-mail: lauseehung@gmail.com

${ }^{a}$ Professor, E-mail: ckng@feng.unimas.my

${ }^{\mathrm{b}}$ Senior Lecturer, E-mail: kmtay@feng.unimas.my 